\title{
The validity of shortened multiple-breath washout testing using sulfur hexafluoride in the assessment of patients with COPD
}

\author{
Enya Daynes $\mathbb{B}^{1}$, Neil Greening ${ }^{1}$, John Owers-Bradley ${ }^{2}$, Sally J. Singh ${ }^{1}$ and Salman Siddiqui ${ }^{3}$
}

${ }^{1}$ Centre of Exercise and Rehabilitation Sciences, NIHR Leicester Biomedical Research Centre - Respiratory, Leicester, UK. ${ }^{2}$ School of Physics and Astronomy, University of Nottingham, Nottingham, UK. ${ }^{3}$ NIHR Leicester Biomedical Research Centre - Respiratory, Leicester, UK.

Corresponding author: Enya Daynes (enya.daynes@uhl-tr.nhs.uk)
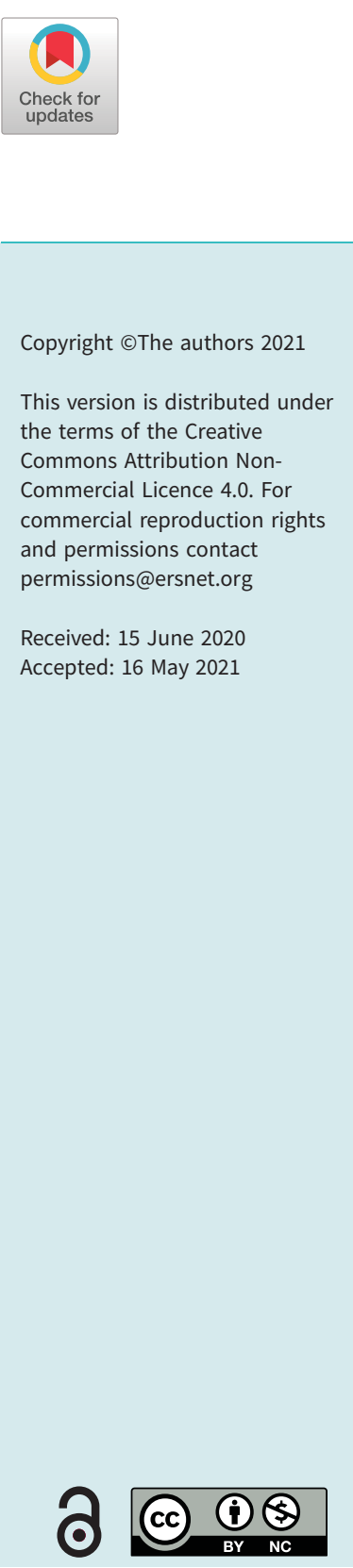

Shareable abstract (@ERSpublications)

It is feasible to shorten the LCI to a $1 / 20$ th washout in patients with COPD, and this leads to a time saving of $32 \%$ and therefore may allow for easier use in clinical settings https://bit.ly/3v9UsXd

Cite this article as: Daynes E, Greening N, Owers-Bradley J, et al. The validity of shortened multiplebreath washout testing using sulfur hexafluoride in the assessment of patients with COPD. ERJ Open Res 2021; 7: 00379-2020 [DOI: 10.1183/23120541.00379-2020].

\section{Abstract}

Introduction COPD affects the small airways and is associated with ventilation heterogeneity. There are little data on the multiple-breath washout (MBW) in patients with COPD, particularly the variability over 8 weeks, using a shortened sulfur hexafluoride $\left(\mathrm{SF}_{6}\right)$ washout. This work evaluated the repeatability of the lung clearance index $(\mathrm{LCI})_{1 / 40}$ and $\mathrm{LCI}_{1 / 20}$ among subjects with COPD and compared to spirometry and clinical markers.

Methods The MBW was performed on patients with COPD to determine ventilation heterogeneity globally (LCI), at conductive $\left(\mathrm{S}_{\text {cond }}\right)$ and acinar $\left(\mathrm{S}_{\text {acin }}\right)$ levels. The LCI was repeated in triplicate and measured at a traditional $1 / 40$ th washout and retrofitted to a shortened $1 / 20$ th end tidal $\mathrm{SF}_{6}$ concentration washout. Tests were repeated after $20 \mathrm{~min}$ and 8 weeks to determine within and between visit repeatability and compared with spirometry.

Results Eighty-four subjects were recruited to perform LCI and spirometry with 20 subjects performing the repeatability protocol. There were weak correlations between forced expiratory volume in $1 \mathrm{~s}\left(\mathrm{FEV}_{1}\right)$ per cent predicted and $\mathrm{LCI}_{1 / 40 \text { th }} \mathrm{r}=-0.311$ ( $\left.\mathrm{p}=0.02\right)$, and $\mathrm{LCI}_{1 / 20 \text { th }} \mathrm{r}=-0.40 \quad(\mathrm{p}<0.01)$. The LCI demonstrated excellent within and good between visit repeatability for both a 1/40th and 1/20th washout (intraclass correlation coefficient (ICC) $\geqslant 0.80$ ). There was a statistically significant strong correlation between $\mathrm{LCI}_{1 / 40 \text { th }}$ and a shortened $\mathrm{LCI}_{1 / 20}$ of $0.86(\mathrm{p}<0.01)$.

Conclusions The LCI is repeatable within and between visits. There are weak correlations with measures of spirometry. A shortened $\mathrm{LCI}_{1 / 20 \text { th }}$ starting concentration correlates highly with a $1 / 40$ th washout, which may encourage clinical use.

\section{Introduction}

COPD is a small airways disease leading to gas mixing abnormalities [1]. The multiple-breath washout (MBW) test is used to determine the lung clearance index (LCI), and further analysis of phase III slopes may enable partitioning of conductive and acinar zone heterogeneities $\left(\mathrm{S}_{\text {cond }} / S_{\text {acin }}\right)$ [2, 3]. A recent study identified that LCI measured with sulfur hexafluoride $\left(\mathrm{SF}_{6}\right)$ as a tracer gas was sensitive to early disease in patients with COPD that had preserved spirometry [4]. It is therefore possible that LCI is a useful early disease and therapeutic biomarker in COPD.

Recently standardisation of the MBW technique has been reported to enable comparison across clinical studies and standardised reporting [5]. LCI is calculated by measuring the number of turnovers of functional residual capacity (FRC) to reduce the end tidal tracer gas concentration (Cet) to 1/40th of its original concentration. The termination point of the test requires at least three consecutive breaths to have a Cet $<1 / 40$ th. Although the 1/40th stopping point is traditionally deployed in testing, its choice is arbitrary and often mandates longer testing times in patients with established lung disease, e.g. COPD. 
Given that, the Association of Respiratory Technology and Physiology (ARTP, UK based) suggests that high-quality spirometry takes $\sim 20 \mathrm{~min}$, a shortened LCI to $\leqslant 20 \mathrm{~min}$ may be more appealing [6]. The combination of reduced testing time and greater disease sensitivity could render LCI a clinically viable tool in COPD management. Additionally, the use of the LCI as a primary outcome in COPD interventional studies is limited and could be increased with knowledge of required sample sizes.

A recent study in cystic fibrosis identified that shortening of the LCI led to significant test time saving without compromising the accuracy of the test [7]. $\mathrm{LCI}_{1 / 20 \text { th }}$ was shown to have high intra-test repeatability and accuracy for cystic fibrosis discrimination from health when compared to $\mathrm{LCI}_{1 / 40 \text { th }}$. Similar results have been observed in asthma, where $\mathrm{LCI}_{1 / 20 \text { th }}$ demonstrated a similar within-visit coefficient of variation and diagnostic sensitivity compared to the 1/40th test [8].

We hypothesised that a shortened (1/20th) LCI is as repeatable as conventional LCI (1/40th) both within visit and over 8 weeks in patients with established COPD. Eight-week repeatability was used to generate sample size calculation for experimental medicine studies with $\mathrm{LCI}_{1 / 40 \text { th }}$ as an outcome measure. Furthermore, we aimed to explore the correlation of $\mathrm{LCI}_{1 / 20 \text { th }}$ with clinical markers of COPD, specifically Global Initiative for Chronic Obstructive Lung Disease (GOLD) stage spirometry and COPD Assessment Test (CAT) scores.

\section{Methods}

This study was performed at the NIHR Biomedical Research Centre - Respiratory at the University Hospital of Leicester, UK during 2017-2018. This study was part of a larger feasibility and randomised controlled trial (ISRCTN 81979106 and ISRCTN45695543); ethical approval was obtained by the National Health Service Health Research Authority and Local Research Ethics Committee (17/EM/0156). Participants were recruited through research databases and outpatient COPD and Pulmonary Rehabilitation clinics and were representative of patients with COPD attending secondary care. Patients were $\geqslant 40$ years old with a confirmed diagnosis of COPD in accordance with the 2017 Global Initiative for Lung Disease (GOLD) recommendations [12]. This includes a current smoking history of $\geqslant 10$ pack-years with at least one of the following symptoms: productive cough, dyspnoea or wheeze and evidence of airflow obstruction (forced expiratory volume in $1 \mathrm{~s}\left(\mathrm{FEV}_{1}\right)$ /forced vital capacity (FVC) ratio of <0.7). Patients were included irrespective of their smoking status.

MBW was measured using a modified photoacoustic Innocor ${ }^{\circledR}$ device as manufactured by Innovision ApS, Denmark. This is an open circuit using $0.2 \% \mathrm{SF}_{6}$ during wash-in and room air for the washout phase. The MBW was performed in line with the European Respiratory Society/American Thoracic Society recommendations [5]. In upright sitting, participants were instructed to perform tidal breathing (7-12 $\mathrm{mL}$ per $\mathrm{kg}$ of their weight) for the duration of the test with a nose clip in situ and performed in triplicate. The procedure was performed at baseline (V1) repeated after a 20-min interval (V2) to determine within-visit repeatability and again after an 8-week interval (V3) to determine between-visit repeatability of the MBW. Analysis was performed using a custom MATLAB algorithm (software version Open_analysis_12 m) updated November 25, 2016. A modification was made to the coding to identify when the concentration reached $<1 / 20$ th starting concentration over three tidal breaths. The phase III slope analysis was programmed between 1.5 and 6 turnovers of FRC to enable a reliable calculation of $\mathrm{S}_{\text {cond; }}$; this has been previously reported in the literature [9]. At least two tests must be within $10 \%$ of the highest FRC to be included in the analysis. The LCI is calculated as the cumulative exhaled volume/FRC. The mean averages of the included tests were used at each time point. Phase III slope analysis was performed to determine the $S_{\text {cond }}$ and $S_{\text {acin }}$ measurements with $S_{\text {cond }}$ calculated by a pooled slope analysis, using the pooled breaths from each included test per time point (two or three tests depending on FRC variability). A previously reported two-compartment lung washout model was used to generate $\mathrm{LCI}_{\text {vent }}$ (ventilation) and $\mathrm{LCI}_{\mathrm{ds}}$ (dead space) with a view to understanding the relative contribution of these factors to LCI. The model outputs report proportional increase in LCI above its ideal value due to specific ventilation inequality $\left(\mathrm{LCI}_{\mathrm{vent}}\right)$ or due to increased respiratory dead space $\left(\mathrm{LCI}_{\mathrm{ds}}\right)$ [9, 13]. Shortening the LCI to a 1/20th washout was performed in the data analysis phase for direct test comparisons; this was performed using a customised MATLAB algorithm and used a predefined 1/20th cut-off (of three consecutive breaths) and corresponding FRC from the 1/20th washout.

Spirometry was performed according to the European Respiratory Society/American Thoracic Society recommendations using a Vitalograph Alpha touch spirometer. The 2012 Global Lung Initiative reference values were used [14]. Spirometry was performed as a screening tool prior to the first MBW and again on participants' second visit prior to their final MBW, $1 \mathrm{~h}$ before MBW testing. The peak test was used for the analysis as this is an effort-dependent test. Participant's height and weight were collected in order to 
calculate spirometry per cent predicted measures and body mass index (BMI). LCI and spirometry were performed at the same time of day across the two visits.

Data were analysed using SPSS statistics for Windows V25 (IBM, New York, USA) (version 24). Correlations between LCI and spirometry were performed using Pearson's correlations. Repeatability of the LCI and spirometry was determined by using Cronbach's $\alpha$ and is presented as an intraclass correlation coefficient (ICC). ICC was calculated using a two-way mixed effects analysis of absolute agreement for single raters. A correlation coefficient of $<0.5$, between 0.5 and 0.74 , between 0.75 and 0.9 and values above 0.9 are considered poor, moderate, good and excellent repeatability, respectively [15]. BlandAltman plots were derived and assessed for systematic bias [16, 17]. A one-way ANOVA with post hoc tests (Tukey's) was used to assess the LCI and GOLD staging and a negative binomial regression was used in the exacerbation analysis. There was no formal power calculation for this study; however, a minimum of 20 subjects were required for the repeatability data analysis.

Results

Clinical study populations

Eighty-four subjects with COPD were recruited to complete $\mathrm{MBW}_{-} \mathrm{SF}_{6}$, spirometry and CAT scores. A sub-set of 20 subjects completed repeatability analysis of $\mathrm{LCI}_{1 / 40 \text { th }}$ and $\mathrm{ICI}_{1 / 20 \text { th }}$ over 8 weeks (table 1 ). Clinical characteristics of the study populations are outline in table 1.

\section{$\mathrm{LCl}$ repeatability}

Nineteen out of 20 completed the protocol and were eligible for repeatability analysis, and 80 out of 84 tests in the correlation cohort were suitable for analysis (5\% fell outside of acceptable $\pm 10 \%$ variation of FRC). Baseline characteristics can be seen in table 1 . The $\mathrm{LCI}_{1 / 20 \text { th }}$ showed good repeatability both within and between visits (8-week) (ICC $\geqslant 0.8$ ). This was comparable to $\mathrm{LCI}_{1 / 40 \text { th }}$ and $\mathrm{FEV}_{1}$ (table 2 ). In addition, the between visit repeatability of $\mathrm{LCI}_{1 / 20 \text { th }}$ was comparable to spirometry. $\mathrm{S}_{\text {acin }}$ phase III slope measures were also repeatable (ICC $\geqslant 0.80$ ) within and between visit; however, $S_{\text {cond }}$ measures were not repeatable over 8 weeks in keeping with previous observations in asthma [9]. There is a poor ICC for LCI vent $_{\text {and }}$ $\mathrm{LCI}_{\mathrm{ds}}$ between visits $\left(0.10\right.$ and 0.05 , respectively). The $\mathrm{LCI}_{\mathrm{vent}}$ and $\mathrm{LCI}_{\mathrm{ds}}$ were calculated to assess the proportionate changes in ventilation and dead space. The measured $\mathrm{LCI}_{\mathrm{ds}}$ was 1.37 (0.32) and $\mathrm{LCI}_{\text {vent }} 1.59$ (0.23). There was a $2 \%$ decrease and $11 \%$ increase in $\mathrm{LCI}_{\mathrm{vent}}$ and $\mathrm{LCI}_{\mathrm{ds}}$, respectively. The coefficient of variation for the $\mathrm{LCI}_{1 / 40 \text { th }}$ was $15 \%$ and for the $\mathrm{LCI}_{1 / 20 \text { th }}$ was $12 \%$. The mean per cent change within-visit (V1 and V2) was $-1.12 \%$ for the $\mathrm{LCI}_{1 / 40 \text { th }}$ and $-2.18 \%$ for the $\mathrm{LCI}_{1 / 20 \text { th }}$ and $\mathrm{r}$ between visit (V1 and V3) was $-1.39 \%$ for $\mathrm{LCI}_{1 / 40 \text { th }}$ and $-3.24 \%$ for $\mathrm{LCI}_{1 / 20 \text { th. }}$.

Bland-Altman plots demonstrating potential proportionate systematic bias for $\mathrm{LCI}_{1 / 40 \text { th }}$ and $\mathrm{LCI}_{1 / 20 \text { th }}$ are presented in figure 1.

\section{TABLE 1 Clinical characteristics of study populations}

\begin{tabular}{|c|c|c|}
\hline & All subjects & Repeatability cohort \\
\hline Subjects $\mathrm{n}$ & 84 & 20 \\
\hline Gender male:female & $62: 22$ & $15: 5$ \\
\hline Age years & $70 \pm 7.3$ & $69 \pm 7$ \\
\hline $\mathrm{FEV}_{1} \%$ predicted & $51 \pm 17$ & $50 \pm 15$ \\
\hline $\mathrm{FEV}_{1} \mathrm{~L}$ & $1.36 \pm 0.53$ & $1.35 \pm 0.43$ \\
\hline FVC L & $2.70 \pm 0.86$ & $2.57 \pm 0.55$ \\
\hline $\mathrm{FEV}_{1} / \mathrm{FVC}$ ratio & $0.49 \pm 0.13$ & $0.52 \pm 0.13$ \\
\hline \multirow[t]{3}{*}{ GOLD spirometry stage } & GOLD 1: 2 & GOLD 1: 0 \\
\hline & GOLD 2-3: 69 & GOLD 2-3: 17 \\
\hline & GOLD 4: 13 & GOLD 4:3 \\
\hline CAT score & $18.64 \pm 7.48$ & $21.14 \pm 7.15$ \\
\hline mMRC score & $2.85 \pm 0.83$ & $2.93 \pm 0.75$ \\
\hline BMI kg $\cdot \mathrm{m}^{-2}$ & $29 \pm 7$ & $29 \pm 7$ \\
\hline Smoker current:ex & $9: 75$ & $1: 19$ \\
\hline $\begin{array}{l}\text { Baseline characteristics } \\
1 \mathrm{~s} \text {; FVC: forced vital ca } \\
\text { Assessment Test score; } n\end{array}$ & $\begin{array}{l}\text { ess otherwise } \\
\text { ative for Chror } \\
\text { esearch Counc }\end{array}$ & $\begin{array}{l}\text { expiratory volume in } \\
\text { ase; CAT score: COPD } \\
\text { ody mass index. }\end{array}$ \\
\hline
\end{tabular}


TABLE 2 Intraclass correlation coefficients for within and between visit measures of the lung clearance index

\begin{tabular}{|c|c|c|c|c|c|}
\hline & Mean \pm sD V1 & Mean \pm sD V2 & Within visit ICC $20 \mathrm{~min}$ & Mean \pm sD V3 (between visit) & Between visit ICC 8 weeks \\
\hline FEV $_{1}$ & $1.36 \pm 0.47$ & $1.42 \pm 0.46$ & & $1.35 \pm 0.43$ & 0.92 \\
\hline $\mathrm{FEV}_{1} \%$ & $51.45 \pm 14.86$ & $52.94 \pm 16.71$ & & $52.94 \pm 16.71$ & 0.92 \\
\hline $\mathrm{FEF}_{25-75}$ & $0.58 \pm 0.36$ & $0.62 \pm 0.26$ & & $0.62 \pm 0.26$ & 0.64 \\
\hline FVC L & $2.58 \pm 0.63$ & $2.86 \pm 0.73$ & & $2.57 \pm 0.55$ & 0.74 \\
\hline $\mathrm{LCl}_{1 / 40 \text { th }}$ & $11.24 \pm 2.25$ & $11.11 \pm 1.90$ & 0.92 & $11.42 \pm 2.03$ & 0.88 \\
\hline $\mathrm{FRC}_{1 / 40 \text { th } \mathrm{L}}$ & $3.51 \pm 1.11$ & $3.19 \pm 0.93$ & 0.96 & $3.50 \pm 0.89$ & 0.92 \\
\hline $\mathrm{S}_{\text {cond }}$ & $0.06 \pm 0.11$ & $0.06 \pm 0.15$ & 0.94 & $0.04 \pm 0.03$ & 0.52 \\
\hline $\mathrm{S}_{\mathrm{acin}}$ & $0.784 \pm 0.46$ & $0.84 \pm 0.59$ & 0.95 & $0.698 \pm 0.40$ & 0.80 \\
\hline $\mathrm{LCl}_{\mathrm{ds}}$ & $1.29 \pm 0.36$ & $1.27 \pm 0.21$ & 0.47 & $1.43 \pm 0.38$ & 0.05 \\
\hline $\mathrm{LCl}_{\text {vent }}$ & $1.54 \pm 0.31$ & $1.50 \pm 0.25$ & 0.80 & $1.51 \pm 0.20$ & 0.10 \\
\hline $\mathrm{LCl}_{1 / 20 \text { th }}$ & $8.59 \pm 1.43$ & $8.40 \pm 1.27$ & 0.96 & $8.53 \pm 1.18$ & 0.80 \\
\hline $\mathrm{FRC}_{1 / 20 \text { th } \mathrm{L}}$ & $3.40 \pm 1.05$ & $3.55 \pm 1.01$ & 0.90 & $3.38 \pm 1.01$ & 0.91 \\
\hline
\end{tabular}

$\mathrm{n}=19$. Intraclass correlation coefficient (ICC) measured by Pearson's correlation coefficient. $\mathrm{FEV}_{1}$ : forced expiratory volume in $1 \mathrm{~s}$; $\mathrm{FEF}_{25-75}$ : forced expiratory flow at at $25-75 \%$ of FVC; FVC: forced vital capacity; LCI: lung clearance index; FRC: functional residual capacity; Scond: convection-dependent ventilation heterogeneity, $\mathrm{S}_{\text {acin }}$ : diffusion-convection-dependent ventilation heterogeneity. $1 / 40 \mathrm{th}=\mathrm{FRC}$ or $\mathrm{LCl}$ calculated at traditional stopping point. $1 / 20$ th=FRC or LCl calculated at truncated stopping point of $1 / 20$ th of the end tidal wash-in concentration over three consecutive breaths.

LCI ${ }_{1 / 20 \text { th }}$ did not demonstrate significant systematic measurement bias both within visit and between visit over 8 weeks (within-visit bias slope (mean $\pm \mathrm{SD}$ ) $0.19 \pm 0.6$ ), $\mathrm{p}=0.13, \mathrm{R}^{2}=0.16$, between visit bias slope (mean \pm SD) $0.29 \pm 0.98, \mathrm{p}=0.85, \mathrm{R}^{2}<0.01$ ). Numerical bias slopes were small and similar for $\mathrm{LCI}_{1 / 20 \text { th }}$ and $\mathrm{LCI}_{1 / 40 \text { th }}$ within and between visits $\left(\mathrm{LCI}_{1 / 40 \text { th }}\right.$ within-visit bias slope (mean $\pm \mathrm{sD}$ ) $-0.07 \pm 1.40, \mathrm{p}=0.44$, $\mathrm{R}^{2}=0.04$, between-visit bias slope (mean $\pm \mathrm{SD}$ ) $0.15 \pm 1.35, \mathrm{p}=0.56, \mathrm{R}^{2}=0.02$ ).

Relationship between $\mathrm{LCl}_{1 / 40 \text { th }}$ and $\mathrm{LCl}_{1 / 20 \text { th }}$

In keeping with an earlier study [4] in COPD, $\mathrm{LCI}_{1 / 40 \text { th }}$ and $\mathrm{LCI}_{1 / 20 \text { th }}$ were highly correlated with numerically smaller values for $\mathrm{LCI}_{1 / 20 \text { th }}$, Pearson correlation of $0.86(\mathrm{p}<0.01)$, 1. The $\mathrm{LCI}_{1 / 40 \text { th }}$ and $\mathrm{LCI}_{1}$, 20th Bland-Altman plots show a bias slope of $3.02(0.70)$. There were statistically significant proportionate biases when comparing $\mathrm{LCI}_{1 / 40 \text { th }}$ and $\mathrm{LCI}_{1 / 20 \text { th }}$; however, the magnitude of this was small $(\mathrm{p}<0.01$, $\mathrm{R}^{2}=0.26$ ) (figure 2).

Testing time and clinical feasibility of $\mathrm{LCl}_{1 / 20 t h}$

The mean \pm SD test time for a $1 / 40$ th washout test was $3.40 \pm 1.55 \mathrm{~min}(204 \pm 93$ seconds) and $10.22 \pm 4.63$ min (613 \pm 278 seconds) if performed in triplicate. A shortened washout to $1 / 20$ th starting concentration has a mean \pm SD test time of $2.33 \pm 1.02$ min $(140 \pm 61$ seconds) or $7.00 \pm 3.05$ min $(420 \pm 183)$ if performed in triplicate, using a $1 / 20$ th washout results in a shortened test of $31.5 \%$ than a $1 / 40$ th washout test. All patients with COPD were able to perform a triplicate LCI test; however, $n=1$ was unsuitable for analysis as it was outside the accepted variance of $10 \%$.

\section{Sample size estimate for experimental medicine studies}

There is no known minimal clinically important difference (MCID) available for the LCI in COPD. We have therefore estimated the treatment effect with LCI as an outcome measure in an experimental medicine study based on mean group changes above the reported limits of agreement.

Table 3 shows the sample size estimates based on superiority at $80 \%$ and $90 \%$ powered (ß) and a 5\% Type I error $(\alpha)$ using standard deviations seen in our within-visit and 8-week repeatability cohort study (2.25 and 2.03, respectively). The power calculations were based on the mean change above the limits of agreements. $\sigma$ is the standard deviation.

$$
n=\frac{f(\alpha / 2, \beta)^{2} \sigma^{2}}{(\text { mean change })^{2}}
$$

Clinical association studies of $\mathrm{LCI}_{1 / 20 t h}$ and spirometry GOLD stage

$\mathrm{LCI}_{1 / 20 \text { th }}$ and $\mathrm{LCI}_{1 / 40 \text { th }}$ were associated with COPD GOLD spirometry stage (figure 3, table 4). There were no significant differences between GOLD staging and $\mathrm{S}_{\text {cond }}$ or $\mathrm{S}_{\text {acin }}$. 
Difference versus average $\mathrm{LCl}_{1 / 40 \text { th }}$ within visit

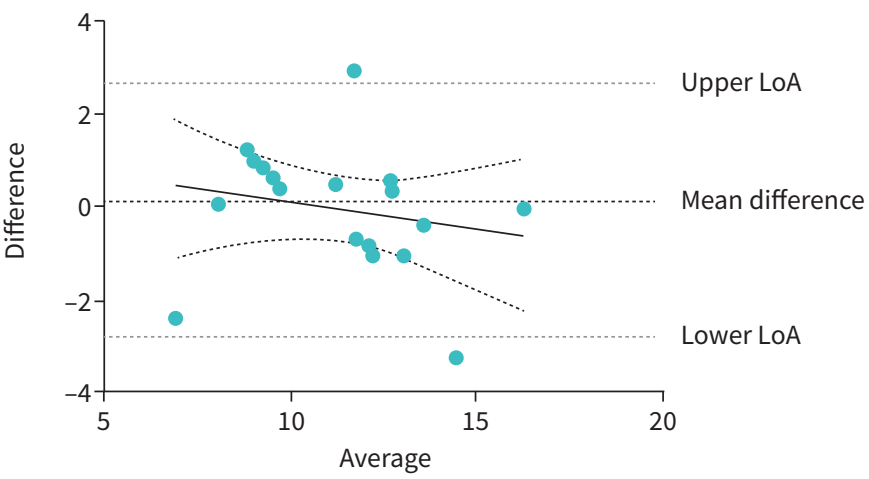

Difference versus average $\mathrm{LCl}_{1 / 40 \text { th }}$ between visit

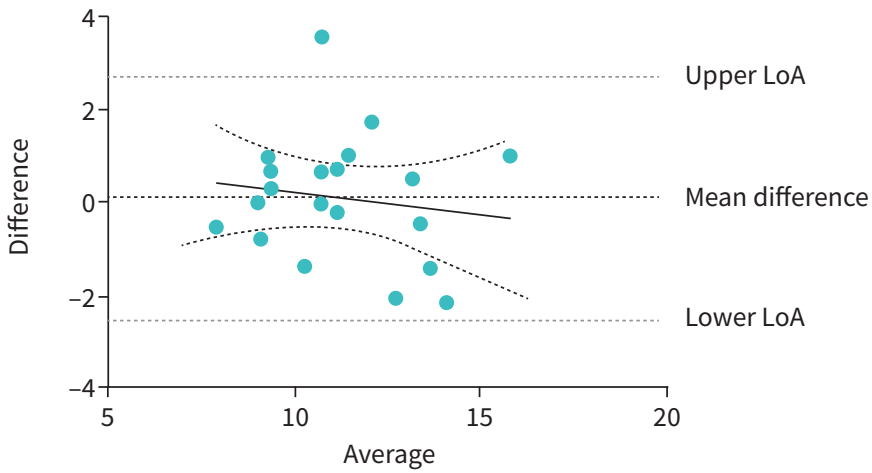

Difference versus average $\mathrm{LCl}_{1 / 20 \text { th }}$ within visit

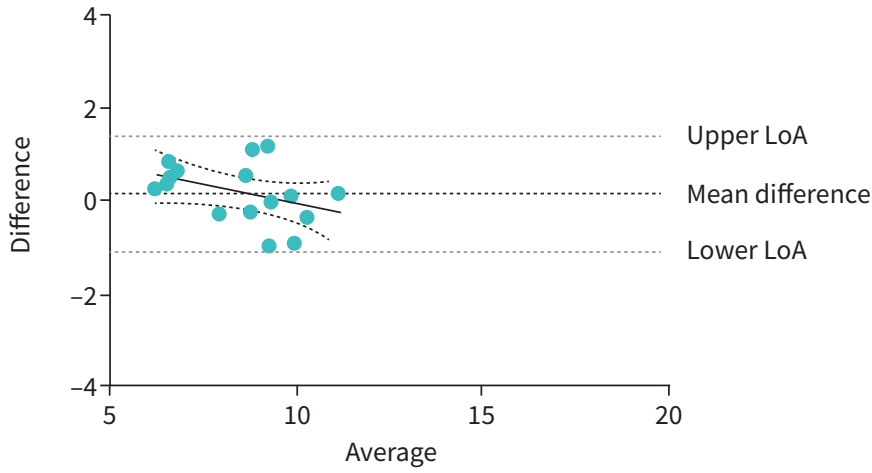

Difference versus average $\mathrm{LCl}_{1 / 20 \text { th }}$ between visit

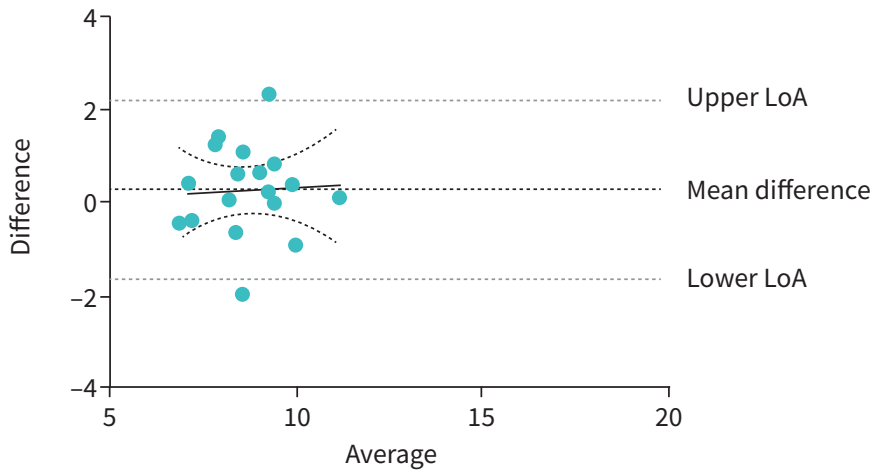

FIGURE 1 Bland-Altman plots for lung clearance index $(\mathrm{LCl})_{1 / 40 \text { th }}$ and $\mathrm{LCl}_{1 / 20 \text { th }}$ within and between visits. Bland-Altman plots for $\mathrm{LCl} \mathrm{I}_{1 / 40 \text { th }}$ within visit repeatability, $\mathrm{LCl}_{1 / 20 \text { th }}$ within visit repeatability, $\mathrm{LCl}_{1 / 40 \text { th }}$ between visit ( 8 week) repeatability and $\mathrm{LCl}_{1 / 20 \text { th }}$ between visit (8 week) repeatability. LoA: limits of agreement $95 \%$ confidence interval.

Correlation matrix of $\mathrm{LCl}_{1 / 20 t h}$ and other physiological indices

There were statistically significant strong correlations between $\mathrm{LCI}_{1 / 20 \text { th }}$ and $\mathrm{LCI}_{1 / 40 \text { th }}$, which remained significant with multivariate linear regression. There were weak correlations with $\mathrm{LCI}_{1 / 20 \text { th }}, \mathrm{LCI}_{1 / 40 \text { th }}$ and spirometry properties, which were statistically significant ( $\mathrm{p}<0.01$ and $\mathrm{p}=0.02$, respectively) (table 5 ).

LCI /20th association with CAT, MMRC symptoms scores and exacerbation frequency

There were no significant differences for the $\mathrm{LCI}_{1 / 20 \text { th }}$ stratified by their mMRC score or CAT total score. There were no significant correlations between symptoms (mMRC or CAT) with $\operatorname{LCI}_{1 / 20 \text { th }}(0.21$ and 0.20 , respectively). Participants were stratified on severity of MRC $(>3)$ and CAT $(>10)$ and analysed using receiver operating characteristics. The area under the curve stratified by MRC score for the $\mathrm{LCI}_{1 / 20 \text { th }}$ was $0.50, \mathrm{LCI}_{1 / 40 \text { th }} 0.41$ and $\mathrm{FEV}_{1} \%$ predicted 0.61 . The area under the curve for the CAT total score for $\mathrm{LCI}_{1 / 20 \text { th }}$ is $0.35, \mathrm{LCI}_{1 / 40 \text { th }} 0.45$ and $\mathrm{FEV}_{1} \%$ predicted 0.73 . Negative binomial regression demonstrated no association between exacerbation frequency in the year prior to the first study visit and LCI (data not shown).

\section{Discussion}

This study explores the use of the LCI across all severities of patients with COPD as the use of a potential outcome measure. The aims of this study were to understand the repeatability of using a shortened MBW within a study visit and over 8 weeks. Testing was conducted across the range of COPD severities without test failure or dropout. The performance of the MBW can be affected if patients experience a chronic, irritable cough, and this may explain some of the tests that were excluded from the analysis; this was also the case in patients with bronchiectasis [10].

In order to use the LCI as a potential therapeutic outcome measure, it is necessary to understand repeatability. The repeatability was assessed following a 20-min rest period within visit and after 8 weeks, as these are 


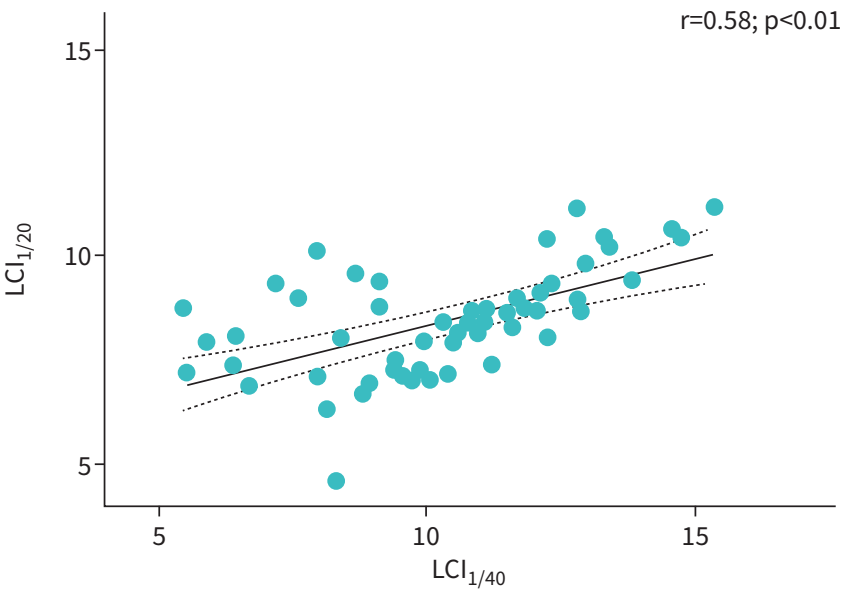

FIGURE 2 Correlations between lung clearance index $(\mathrm{LCl})_{1 / 20 \text { th }}$ and $\mathrm{LCl}_{1 / 40 \text { th }}$ washout test. Correlations between lung clearance index and $1 / 40$ th and $1 / 20$ th washout concentrations. Plotted with a line of best fit and the confidence intervals (dotted line).

common time points in therapy treatment. The use of the $\mathrm{LCI}_{1 / 40 \text { th, }} \mathrm{LCI}_{1 / 20 \text { th, }}, \mathrm{FRC}, \mathrm{S}_{\text {cond }}$ and $\mathrm{S}_{\text {acin }}$ were all highly repeatable after 20 min of rest. The $\mathrm{LCI}_{1 / 40 \text { th }}$, LC $\mathrm{I}_{/ 20 \text { th }}$, FRC and $\mathrm{S}_{\text {acin }}$ were highly repeatable over an 8-week period; however, there was moderate-poor repeatability of the $\mathrm{S}_{\text {cond }}$ and $\mathrm{LCI}_{\mathrm{vent}}$ over this time period. This may be explained by the instrument noise associated with this measure or variability in the condition and is similar to results seen in patients with asthma [9]. The $\mathrm{LCI}_{1 / 20 \text { th }}$ demonstrated an increased variability compared to $\mathrm{LCI}_{1 / 40 \text { th }}$ when comparing relative change and coefficient of variance between visits. This may be due to the reduced testing time that limits the assessment of the smaller airways and that could be assessed on a 1/40th washout; however, the ICC for both the $\mathrm{LCI}_{1 / 40 \text { th }}$ and $\mathrm{LCI}_{1 / 20 \text { th }}$ were similar at 8 weeks $(0.88$ and 0.80 , respectively). The use of LCI using a nitrogen washout has demonstrated an increased LCI in patients with mild to moderate COPD, which is comparable to the data shown in this work [4]. Compared to healthy control literature on LCI, the patients in this study demonstrated an increased LCI; however, this literature is predominantly in a younger population than this study. GRILLO et al. [10] demonstrated an LCI of 7.36 \pm 0.99 in patients with bronchiectasis a mean \pm SD age of $49.9 \pm 13.42$. Age can increase the LCI, which may have contributed to the increased LCI in this study; however, it is likely that the level of airflow obstruction predominantly contributed to this increase $[18,19]$. The coefficient of variation of the $\mathrm{LCI}_{1 / 40 \text { th }}$ was higher than reported in the literature, which could be due to the inclusion of all disease severities from GOLD stage one through to four [4]. Analysis of the $\mathrm{LCI}_{\mathrm{vent}}$ and $\mathrm{LCI}_{\mathrm{ds}}$ using a previously reported twocompartment model enabled exploration of ventilation and dead space contributions to ventilation heterogeneity [13]. We observed poor within and between visit repeatability of these measures. There are a number of plausible explanations for this observation. A two-compartment model assumes that the LCI can be described by the weighted sum of two exponential decay rate constants, whereas in COPD there may be many heterogeneous compartments all emptying according to their own rate constants due to altered local parenchymal tissue mechanics as a consequence of emphysema, small airways disease and mucous plugging. The poor within

TABLE 3 Sample size calculation for within and between visit (V) study designs

\begin{tabular}{lcccc} 
Change in LCI & \multicolumn{2}{c}{$\begin{array}{c}\text { Within visit limits of agreement } \\
(\mathrm{V} 2-\mathrm{V} 1)\end{array}$} & \multicolumn{2}{c}{$\begin{array}{c}\text { Between visit limits of agreement } \\
\text { (V3-V1) }\end{array}$} \\
\cline { 2 - 4 } & $\mathbf{8 0 \% \text { power }}$ & $\mathbf{9 0 \% \text { power }}$ & $\mathbf{8 0 \% \text { power }}$ & $\mathbf{9 0 \% \text { power }}$ \\
\hline $\mathbf{2 . 4}$ & 15 & 19 & 12 & 16 \\
$\mathbf{2 . 6}$ & 12 & 16 & 10 & 13 \\
$\mathbf{2 . 8}$ & 11 & 14 & 9 & 12 \\
$\mathbf{3 . 0}$ & 9 & 12 & 8 & 10 \\
\hline
\end{tabular}

Estimated sample size calculation using an absolute change in lung clearance index $(\mathrm{LCl})_{1 / 40 \text { th }}$ and the withinand between-visit limits of agreement at $80 \%$ and $90 \%$ power. 

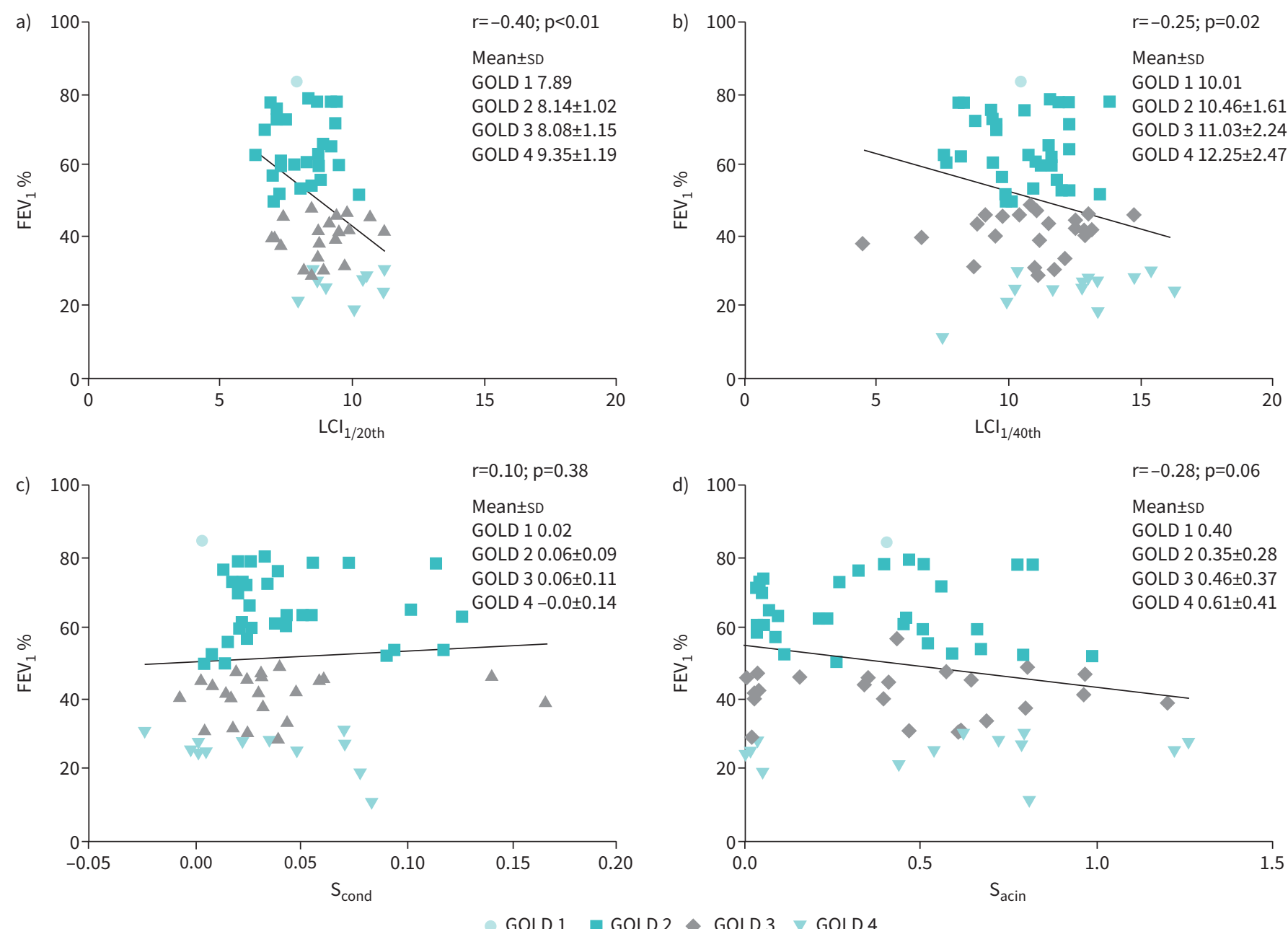

GOLD 1 GOLD 2 GOLD $3 \vee$ GOLD 4

FIGURE 3 Correlations between forced expiratory volume in $1 \mathrm{~s}\left(\mathrm{FEV}_{1}\right) \%$ predicted and a) lung clearance index $\left.\left(\mathrm{LCl}_{1 / 20 \text { th }}, \mathrm{b}\right) \mathrm{LCl} \mathrm{L}_{1 / 40 \text { th }}, \mathrm{C}\right) \mathrm{S}_{\mathrm{cond}}$ conducting zone and d) $\mathrm{S}_{\text {acin }}$ acinar zone. Correlations between measures of multiple-breath washout and forced expiratory volume in $1 \mathrm{~s}$ per cent predicted (FEV 1 \%). GOLD: Global Initiative for Chronic Obstructive Lung Disease.

visit repeatability of both $\mathrm{S}_{\text {cond }}$ and $\mathrm{LCI}_{\mathrm{vent}} / \mathrm{LCI}_{\mathrm{ds}}$ in this study could also be explained by stochastic variation of peripheral airway diameters and acinar volumes in COPD or, both [17].

We observed similar repeatability with a 1/20th test to the traditional $1 / 40$ th washout. This was demonstrated through the analysis phase, and therefore testing would need to occur prospectively with a shortened test (stopping at 1/20th of the wash-in gas concentration) to demonstrate the feasibility of

\begin{tabular}{lccccc}
\multicolumn{2}{l}{ TABLE 4} & One-way ANOVA for COPD \\
& GOLD 1 & GOLD 2 & GOLD 3 & GOLD 4 & p-value \\
\hline Subjects n & 2 & 38 & 25 & 14 & \\
$\mathrm{LCl}_{1 / 20 \text { th }}$ & 7.89 & $8.14 \pm 1.02^{\star}$ & $8.08 \pm 1.15$ & $9.35 \pm 1.19^{\star}$ & $<0.01$ \\
$\mathrm{LCl}_{1 / 40 \text { th }}$ & 10.01 & $10.46 \pm 1.6^{\star}$ & $11.03 \pm 2.24$ & $12.25 \pm 2.47^{\star}$ & 0.05 \\
$\mathrm{~S}_{\text {cond }}$ & 0.02 & $0.06 \pm 0.09$ & $0.06 \pm 0.11$ & $-0.01 \pm 0.14$ & 0.31 \\
$\mathrm{~S}_{\text {acin }}$ & 0.40 & $0.35 \pm 0.28$ & $0.46 \pm 0.37$ & $0.61 \pm 0.41$ & 0.16 \\
\hline
\end{tabular}

One-Way ANOVA stratified by disease severity according to the Global Initiative for Chronic Obstructive Lung Disease (GOLD) spirometry strata. $\mathrm{LCl}$ : lung clearance index; $\mathrm{S}_{\text {cond }}$ : conducting zone; $\mathrm{S}_{\text {acin }}$ : acinar zone. *: post hoc tests (Tukey's) between GOLD staging $p<0.05$. 


\section{TABLE 5 Univariate correlation of $\mathrm{LCl}_{1 / 20 \mathrm{th}}$ and physiological indices}

\begin{tabular}{|c|c|c|}
\hline & $\mathrm{LCl}_{1 / 20 \text { th }}$ & $\mathrm{LCl}_{1 / 40 \text { th }}$ \\
\hline $\mathrm{LCl}_{1 / 20 \text { th }}$ & & $0.76^{\star \star}$ \\
\hline $\mathrm{LCl}_{1 / 40 \text { th }}$ & $0.76^{\star \star}$ & \\
\hline $\mathrm{FEV}_{1} \%$ predicted & $-0.40^{\star \star}$ & $-0.25^{\star}$ \\
\hline FVC \% predicted & -0.11 & -0.61 \\
\hline $\mathrm{FEF}_{25-75}$ & -0.43 & -0.36 \\
\hline $\mathrm{S}_{\text {cond }}$ & -0.19 & -0.07 \\
\hline $\mathrm{S}_{\mathrm{acin}}$ & 0.22 & 0.11 \\
\hline
\end{tabular}

performing a shortened washout. The shortening of the multiple-breath washouts may improve its clinical application [6]; however, as this study performed $\mathrm{LCI}_{1 / 20 \text { th }}$ in the analysis phase, the duration of a shortened wash-in has not been explored. The use of a shortened LCI to 1/20th of the starting concentration has been shown to be feasible and repeatable in patients with cystic fibrosis [11]. Shortened LCI tests have been shown to be sensitive in patients with cystic fibrosis compared to healthy controls [7]; however, there are few studies that explore the response of the $\mathrm{LCI}_{1 / 20 \text { th }}$ in COPD or in response to an intervention in these conditions [10].

Power calculations using the repeatability study data have established potential sample sizes for LCI as an end-point which are relatively modest and support the use of LCI as a potential mechanistic or secondary end-point in trials designed to target ventilation heterogeneity. The use of a shortened LCI may reduce the sensitivity, and therefore its application should be justified by the specific research or clinical question [11].

Our study has several potential limitations. The conduction of spirometry an hour before MBW testing may affect the washout, and the impact of this was not explored in this study. There is a weak correlation between MBW measures and clinical outcomes as measured by the CAT, mMRC score and prior exacerbation frequency. The CAT and mMRC are subjective measures of symptoms that are influenced by biological, psychological and social factors. Whilst a worsened LCI may indicate an increased severity of diseased lung and greater disease heterogeneity within the lung, it is not a direct measure of clinical severity and does not account for other factors that can contribute to patient symptoms in COPD. Additionally, reporting of prior exacerbations is variable, and subject to recall bias. Our study did not confirm exacerbation events through primary care record analysis of rescue pack medication. Future work could evaluate the LCI across COPD treatment strata that are classified by both symptoms (mMRC or CAT scores) and exacerbation frequency.

The results of this work support the use of the LCI as a potential tool in experimental medicine studies targeting ventilation heterogeneity in patients with stable COPD. Our demonstration of high within and between visit repeatability of $\mathrm{LCI}_{1 / 20 \text { th }}$ suggests that prospective use of this marker needs to be explored in clinical studies of COPD to identify clinically important treatment effect sizes alongside testing feasibility.

Author contributions: E. Daynes: protocol development, data collection, data analysis and manuscript preparation. N. Greening: protocol development, data analysis and review of the manuscript. J. Owers-Bradley: data analysis and review of the manuscript. S. Singh: protocol development and review of the manuscript. S. Siddiqui: protocol development, data analysis and review of the manuscript.

This study is registered at www.isrctn.com with identifier number ISRCTN45695543. Individual anonymised participant data will be shared between 9 and 36 months after publication for the purposes of meta-analysis. Proposals may be submitted up to 36 months following publication by contacting the corresponding author, after which, you will be redirected to another researcher that may be able to help with your query. Data will be made available upon reasonable request to the authors.

Conflict of interest: E. Daynes reports that Actegy Ltd funds a commercial trial from which the present data were obtained. N. Greening has nothing to disclose. J. Owers-Bradley has nothing to disclose. S. Singh reports that Actegy Ltd funds a commercial trial from which the present data were obtained. S. Siddiqui reports advisory 
boards for and speaker fees from AstraZeneca, GSK, Novartis, Roche, Boehringer Ingelheim, Owlstone Medical, Mundipharma and ERT Medical, outside the submitted work.

Support statement: This study is supported by the National Institute for Health Research (NIHR). The views expressed are those of the author(s) and not necessarily those of the NIHR or the Department of Health and Social Care. This study was funded by Actegy Ltd. Funding information for this article has been deposited with the Crossref Funder Registry.

\section{References}

1 Verbanck S, Schuermans D, Meysman M, et al. Noninvasive assessment of airway alterations in smokers: the small airways revisited. Am J Respir Crit Care Med 2004; 170: 414-419.

2 Verbanck S, Schuermans D, Noppen M, et al. Evidence of acinar airway involvement in asthma. Am J Respir Crit Care Med 1999; 159(5 Pt 1): 1545-1550.

3 Paiva M, Engel L. Model analysis of intra-acinar gas exchange. Respir Physiol 1985; 62: 257-272.

4 Bell AS, Lawrence PJ, Singh D, et al. Feasibility and challenges of using multiple breath washout in COPD. Int J Chron Obstruct Pulmon Dis 2018; 13: 2113-2119.

5 Robinson PD, Latzin P, Verbanck S, et al. Consensus statement for inert gas washout measurement using multiple- and single-breath tests. Eur Respir J 2013; 41: 507-522.

6 Cooper B, Butterfield A. Quality Control in Lung Function Testing. 2019. Available from: www.artp.org.uk.

7 Hannon D, Bradley JM, Bradbury I, et al. Shortened lung clearance index is a repeatable and sensitive test in children and adults with cystic fibrosis. BMJ Open Respir Res 2014; 1: e000031.

8 Trinkmann F, Lenz SA, Schäfer J, et al. Feasibility and clinical applications of multiple breath wash-out (MBW) testing using sulphur hexafluoride in adults with bronchial asthma. Sci Rep 2020; 10: 1527.

9 Gonem S, Corkill S, Singapuri A, et al. Between-visit variability of small airway obstruction markers in patients with asthma. Eur Respir J 2014; 44: 242-244.

10 Grillo L, Irving S, Hansell DM, et al. The reproducibility and responsiveness of the lung clearance index in bronchiectasis. Eur Respir J 2015; 46: 1645-1653.

11 Horsley A. Lung clearance index in the assessment of airways disease. Respir Med 2009; 103: 793-799.

12 Vogelmeier CF, Criner GJ, Martinez FJ, et al. Global strategy for the diagnosis, management, and prevention of chronic obstructive lung disease 2017 report. GOLD Executive Summary. Am J Respir Crit Care Med 2017; 195: 557-582.

13 Gonem S, Scaddin A, Soares M, et al. Lung clearance index in adults with non-cystic fibrosis bronchiectasis. Respir Res 2014; 15: 59.

14 Global Initiative for Chronic Obstructive Lung Disease (GOLD). Global Strategy for the Diagnosis, Management and Prevention of COPD 2015. Available from: www.goldcopd.org. Date last accessed: Mar 2020. Date last updated: Dec 2019.

15 Koo TK, Li MY. A Guideline of selecting and reporting intraclass correlation coefficients for reliability research. J Chiropr Med 2016; 15: 155-163.

16 Bland JM, Altman DG. Statistical methods for assessing agreement between two methods of clinical measurement. Lancet 1986; 1: 307-310.

17 Giavarina D. Understanding Bland Altman analysis. Biochem Med 2015; 25: 141-151.

18 Leary D, Bhatawadekar S, Parraga G, et al. Modelling stochastic and spatial heterogeneity in human airway tree to determine variation in respiratory system resistance. J Appl Physiol 2012; 112: 167-175.

19 Verbanck S, Thompson B, Schuermans D, et al. Ventilation heterogeneity in the acinar and conductive zones of the normal aging lung. Thorax 2012; 67: 789-795. 